\title{
Droplet Breakup in an Asymmetric Bifurcation Consisted of Two Branches
}

\author{
Xiang Wang, Yan Pang, Zhaomiao Liu \\ College of Mechanical Engineering and Applied Electronics Technology, Beijing University of Technology \\ No. 100 Ping Le Yuan Road, Chaoyang District, Beijing, China \\ wxbjut@emails.bjut.edu.cn; pangyan@bjut.edu.cn; lzm@bjut.edu.cn
}

\section{Extended Abstract}

Due to the various advantages, droplet-based microfluidics has found applications in many chemical and biochemical reactions ${ }^{[1]}$ On most occasions, the size and frequency of droplets are two input parameters that determine the outcomes of the droplet-based systems, therefore, they need to be controlled precisely. Droplet breakup is one of the most effective method to tune the droplet size and frequency, and it can be divided into active and passive type. The former are achieved by additional energy fields, such as the heater and electrodes. ${ }^{[2]}$ Complex structures are usually involved and the outcomes may be disturbed. For the passive type, the breakup process depends mainly on the splitting junctions and flow conditions, and the size of daughter droplets could be reduced to very small values and the throughput of droplets is largely increased meanwhile. At the splitting junction, the droplet is stretched with the viscous shear stress of the continuous phase. In order to reduce its surface energy, the droplet would completely flow into one of the branches or split into daughter droplets, determined by the capillary number. ${ }^{[3]}$

In this paper, the passive droplet breakup in an asymmetric bifurcation with two branches is experimentally studied. Sunflower oil and deionized water are used as the continuous and dispersed phase, respectively. In order to separately tune the initial droplet size and droplet velocity, a diluting channel is introduced at the downstream of the generation unit and its influence on droplet generation is confirmed. Effects of the droplet parameters, including the initial droplet length, the droplet velocity, the interfacial tension between the dispersed phase and the continuous phase, on breakup characteristics are analyzed. The splitting ratio is largely influenced by the initial droplet length and the droplet velocity, which proves that the breakup process is an inter-dependent process between the splitting junction and droplets themselves. During droplet breakup, locations of the maximum velocity inside the droplet correspondingly change. Besides, the breakup time is dominated by the velocity of droplets, while only varied a little with the droplet length for short droplets and nearly not dependent on the interfacial tension. Strong similarity is also found in the breakup time between the droplet and the bubble.

\section{Acknowledgements}

The authors are grateful for the support of the National Natural Science Foundation of China (11572013 and 11702007) and the China Postdoctoral Science Foundation (2017M610725).

\section{References}

[1] S. Tasoglu, U. A. Gurkan, S. Wang and U. Demirci, "Manipulating biological agents and cells in micro-scale volumes for applications in medicine," Chem Soc Rev, vol. 42, pp. 5788-5808, 2013.

[2] T. H. Ting, Y. F. Yap, N.-T. Nguyen, T. N. Wong, J. C. K. Chai and L. Yobas, "Thermally mediated breakup of drops in microchannels," Applied Physics Letters, vol. 89, pp. 234101, 2006.

[3] A. M. Leshansky, S. Afkhami, M. C. Jullien and P. Tabeling, "Obstructed breakup of slender drops in a microfluidic T junction,” Physical Review Letters, vol. 108, 264502, 2012. 